\title{
DIVIDING KNOWLEDGE AND SKILLS IN HUMAN HISTORY: A PERSPECTIVE FROM AN ETHNOGRAPHIC STUDY ON THE FOLK SOCIETY
}

\author{
Hideyuki ŌNISHI
}

Doshisha Women's College of Liberal Arts, Japan

\begin{abstract}
This paper illustrates an ethnographic study that covers the practice of non/premodern craftsmanship in folk society, comprehending the mechanism of knowledge and skills which are difficult to visualize and verbalize. This study argues, the technical practice of craft production within folk social society encompasses the materialisation of the invisible/non-verbal implicit knowledge and embodied skills. Moreover, such knowledge and skills are not necessarily limited to non/pre-modern societies, but in contemporary societies after the Industrial Revolution. Finally, it shall be shown that, in Human history, forcing the separation of knowledge and skills, technology had become manualized and verbalized, which lead to an overwhelming majority of society becoming substitutable labourers, eradicating skilled professionals.
\end{abstract}

Key words: body techniques, community of practice, invisible and non-verbal aspect of social practice, sociocultural anthropology, tacit knowledge

\section{INTRODUCTION}

Human beings are categorized within the groupings of Homo Sapiens and yet, are recognized for their diversity of cognition and behaviour due to differences in era, region, and group (Lock, 1993, pp. 135-138). This is certainly because our cognition and behaviour is rooted in the knowledge and skills acquired in a cultural and social environment. Not to mention, these acquired knowledge and skills are commonalities and themes of interest to cultural/social anthropology and psychology/cognitive science. Yet, joint research or constructive discussions amongst the two fields in regards to knowledge and skills has been very limited thus far. Furthermore, it is hard to say that there is sufficient discussion in both fields regarding the historical changes in knowledge and skills.

On the other hand, there are realms in human knowledge and skills where researchers, as well as local participants, find difficult to explain. This complexity is better understood by trying to recollect the difficulty in verbalizing or visualizing practices such as, the enjoyment of a meal or singing a song. If these non-verbal and visual practices are not a

This work was supported by JP19H05735, JP19H05731. And I am deeply grateful to Lise Sasaki for her contribution in translation and academic support.

Correspondence concerning this article should be addressed to Hideyuki Ōnishi, Department of Social System Studies, Faculty of Contemporary Social Studies, Doshisha Women's College of Liberal Arts, Kodo, Kyotanabe, Kyoto 610-0395, Japan (e-mail: honishi@dwc.doshisha.ac.jp). 
fraction of our knowledge and skills, it becomes inevitable to explore this intricate realm. Concentrating on these issues, this paper presents the comprehension of the elaborate knowledge and skills that struggle to be verbalized and visualized. The mentioned knowledge and skills lie in the foundation of non/pre-modern craftsmanship within folk society, as the discussion flows through an ethnographic study. Additionally, this paper will consider the transformation of knowledge and skills in human history by comparing the entities of folk and modern society based on the research outcomes.

\section{RESEARCH BACKGROUND: \\ Perspective and Problem Over Social Practice in Daily life}

Certainly, knowledge and skills are central themes within cultural/social anthropology as well as for psychology/cognitive science. However, cultural/social anthropology and psychology/cognitive science have not always had a supportive relationship within research on knowledge and skills. ${ }^{1}$ Although, from the 1990s, cultural anthropology has received a recursion into the daily, common world after it reflected its excessive epistemology and political criticism based on postmodernism (cf. Tanabe, 1989, 2002; Tanabe \& Matsuda, 2002). Branching from this, this research will highlight ethnographical research: the routines and embodied practices stemming from the daily lives of the local people. Moreover, it will built a close tie with themes and issues of cognitive science.

As Masato Fukushima discusses, there exists a limit to the observations and narratives that ethnographic surveys have traditionally relied upon, especially when trying to understand the customary social practices of the local people (Fukushima, 1993, pp. 12-15). This is because customary social practices are composed of knowledge and skills that are too complex to be visualized or verbalized-not only for the researcher but also for the local participants.

Riding a bike is a typical example (Polanyi, 1969, p. 147; Fukushima, 1993, p. 17) commonly used in this discussion. Regardless of careful observation of the person riding the bike, the information gathered is merely, and no more than, the physicality of operating the bicycle, its simple mechanics and manoeuvred time. Interviewing the actor riding the bicycle, "what is it like to ride a bicycle?" or "how do you ride a bicycle?" will only anticipate a non-specific, mundane answer. To sum up, it is a fact that the results to these interviews do not encompass the entire knowledge of riding a bike - it is clear, that the number of interviews and observations to those riding a bicycle will not reflect on acquiring the skill in ride one.

One way to overcome these limitations is the theories of Michael Polanyi's Tacit knowledge (Polanyi, 1966), Marcel Mauss's The body techniques (techniques du corps;

\footnotetext{
${ }^{1}$ It is safe to say that, a science-oriented psychology/cognitive science that pursues human psychology/ cognition, is a theoretical opponent to cultural/social anthropology which has relativized modern Western society intending to understand various cultures. These views are stereotypical as they ignore the various developments of psychology and cognitive science, but it is also a fact that these prejudices hinder joint research.
} 
Mauss, 1934), and Jean Lave and Etienne Wenger's Community of practice (Lave \& Wenger, 1991). By introducing concepts that have been discussed in psychology/cognitive science, such as Lave and Wenger (1991), attempts are made to interpret the knowledge and skills that support social practice (Fukushima, 1993, 1995). Specifically, these interpretations are based on the premise that it taps into a sphere of non-verbalized tacit knowledge by those who act on social practice. However, this will place tacit knowledge in a black box, incapable of ethnographic analysis. Therefore, to capture this phenomenon, the actors' movement of the body which can be recognized based on the non-verbalized $=$ non-spoken knowledge will be focused and studied. Extending from this, this paper attempts to approach and understand tacit knowledge through material phenomena produced due to practices as body techniques. Furthermore, it is clarified from cases that such non-verbalized tacit knowledge and body techniques can be grasped by repeating unconscious repetitions of daily social life. Places of such practices are described as the community of practice in an ethnography. ${ }^{2}$

The discussions mentioned above is a research program responding to: how to anthropologically illustrate the knowledge and skills that support social practices that are incomprehensible solely through observation and ethnographic interviews. However, there is one issue to be resolved for this program to commence. It is whether to affirm this phenomenon: despite the actor unable to verbalize therefore explain, in reality, the phenomenon is constructed from, and as a result of, bodily movements. Therefore, the phenomenon itself is an outcome of the interventions of knowledge and skills. Phrased differently, there is a risk of falling into the tautology that tacit knowledge is acquired because practices are performed or, practices are performed therefore, tacit knowledge is acquired. To avoid this tautology, it is necessary to clarify whether or not practices can be executed, in other words, whether or not tacit knowledge is achieved explicitly and rationally. The success or failure in enforcing this kind of practise is not upon logically solving the problem, but on persuasive evidence referenced from concrete examples. This is because it is necessary to grasp the events produced by social practices and judge whether or not they reflect the knowledge and skills that are difficult to visualize and verbalize for each specific case.

Regarding the definition of knowledge and skills, there are various views depending on research areas or the position of the researcher which lead to its complex definition. However, for the time being, in this paper, while knowledge can be partially verbalized (Lemonnier, 1992, pp. 81-82), skills, on the other hand, adopting the definition of cultural/social anthropology based on ethnographic surveys, are latent as physical movement and cannot be completely verbalized (Sigaut, 1994, pp. 442, 455-456). Let us clarify this concept with the mentioned practise of riding a bicycle: knowledge is the verbalization of holding the steering wheel and stepping on the pedal with one foot across

\footnotetext{
${ }^{2}$ Needless to say, these methods were selected as one of the solutions based on the ethnographic survey. However, research practices targeting non-verbal knowledge are not limited to ethnographic surveys. As Masato Fukushima (1993, pp. 18-24) argues, efforts are being promoted in various areas of academia for research in non-verbal knowledge such as a wide range of approaches in cognitive science and artificial intelligence research attempting to digitize cognitive and judgment that humans exert in their specialized fields (Ōnishi, 2014, pp. 53-58).
} 
the saddle, and the synchronised physical movement is the skill (Ōnishi, 2014, pp. 33-34). As a side note, following this definition, it is impossible in principle to verify by language whether or not the body has the skills to ride a bicycle. Following the method in this paper, this practice is validated only by confirming the act of riding a bicycle. ${ }^{3}$

\section{MethoD:}

\section{A Tentative Approach to Grasp Knowledge and Skills in the Folk Craft}

Considering the issues mentioned, this paper focuses on the making of pottery, a folk craft of the mountainous community in the northern part of Luzon Island, Philippines, to grasp and understand the invisible and non-verbal knowledge and skills, presenting an example from an ethnographic survey. The survey was conducted from March to July 1996 in a Baranggay named Bila located in Bauko Municipality, the western part of the mountain province composing the Cordillera Administrative Region in northern Luzon (Fig. 1). ${ }^{4}$

To fully study the mechanism of the invisible/non-verbal skills and knowledge, I participated in local pottery making supported by local potters. This exercise emphasizes the experiences shared with local people, extending to the knowledge and skill gained through observation and interviews taken further through bodily participation (Ōnishi, 1998b, pp. 2-3). However, there must be an acknowledgment that,

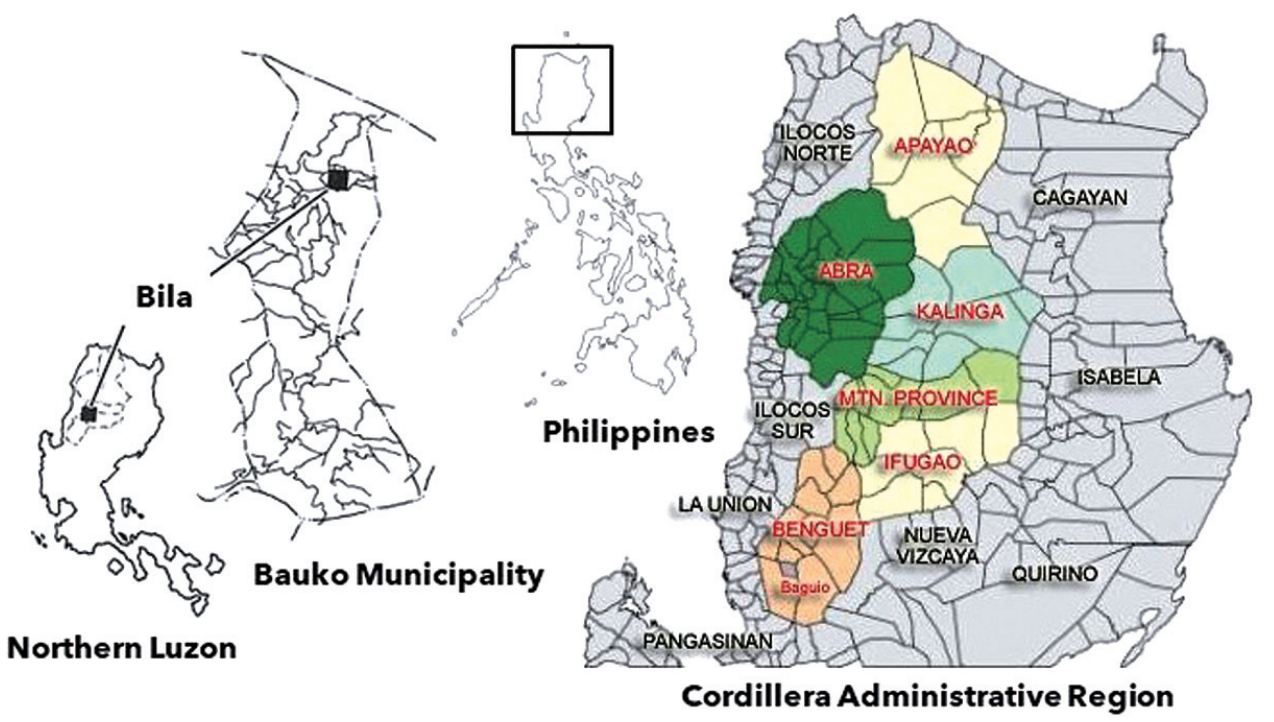

Fig. 1. Sites Concerned This Study

\footnotetext{
${ }^{3}$ Guided by the article's definition, M. Polanyi's tacit knowledge (Polanyi, 1966) should be categorized into skills rather than knowledge, just as scholars such as T. Ingold points out (Ingold, 1993, p. 434). However, these views derive from the modern view of knowledge: knowledge and skills are two separate things, as presented in the conclusion of this article. Therefore, in this paper, we reject the categories such as modern or non/pre-modern and apply a uniform definition to knowledge and skills in any socio-cultural situation.

${ }^{4}$ Details of pottery making in Bila Village and the society of the Cordillera Mountains are described in a few of my publications (Ōnishi, 1998a, 1998b, 2014). Moreover, the time I participated in pottery making was two months from April to May, just before the rainy season.
} 
sharing experiences for a limited time does not result to a complete understanding of the local knowledge and skill. Needless to say, my experience is not an exact trace of the knowledge and skill development of the local people, but merely a limited example of a researcher with cross-cultural experience.

The aim of this survey is only possible founding on this acknowledgement. The comparison of my bodily participation in pottery making with my life experience leads to the possibility of approaching the local knowledge and skills that are difficult to visualize and verbalize. In other words, the research in this paper attempts to actively utilize the variant body the researcher myself carry from the local people and, by experiencing these differences, try to capture the knowledge and skills that are invisible and non-verbalized.

On the other hand, while the discussion above may be agreed upon, there is another issue that cannot be overlooked. It is a question of how to illustrate the knowledge and skills practised in pottery making. This issue is faced not only in ethnographic research but also in artificial intelligence research - the attempt to digitize and computerize the cognitive and judgment systems that humans bring forth in their specialized fields (Fukushima, 1993, pp. 18-24). Such issues are raised only because knowledge and skills are difficult to verbalize. As F. Sigaut points out, knowledge and skills are hidden behind the consequences of actions and are not easily linguistically recorded and communicated (Sigaut, 1994, pp. 442, 445-446). Human beings possess knowledge and skills to control their body's desired action: cooking or driving a car, and so on. Yet, in actuality, linguistic description of these movements and experiences is almost impossible.

Admitting to the statement above will lead to the conclusion that the verbalization and illustration of the overall knowledge and skills of pottery making are impossible, despite acquiring them. Therefore, this research will aim to highlight the mediacy of knowledge or skills rather than concentrate on the factors of the non-verbal. Although clear linguistic communication is difficult, it will present the results achieved by the knowledge and skills practiced in pottery making. To be specific, first, facts observed from the maker's technical practices are studied. If the knowledge and skills that intervene in the milieu of such technical practices are indescribable, reasonings are tackled. From the attempted descriptions, the knowledge and skills that lie in the background of technical practices are surfaced, as well as the clarifications of their characteristics.

\section{Result and Discussion: KNOWledge ANd Skills in the Folk Society}

In the field survey in Bila village, a brief overview of pottery making, including procedures and conventions recognized by the local people, was easily obtained. Even if one does not actively learn pottery making to gain the techniques of fabrication like myself, an easy overview of its mechanism could perhaps be grasped. This may be true in an ordinary ethnographic survey, regardless of the length of period.

However, as I continued to make pottery to acquire knowledge and skills, I realized that there were processes that could never be achieved solely by observation and interviews. An example is the procedure of scraping the inner surface of earthenware. According to local potters, this process sharpens the inner surface with a tool that winds a metal plate in a ring shape to make the pottery thickness even (Fig. 2). When I took part in this practice, it didn't seem like a difficult challenge. Yet, during my participation, a serious issue was faced: the obvious question of how thick the wall should be scraped. This was a very pressing issue as up until then, all seemed to be going well. It was when I began the scraping process that I sensed an embarrassment as I didn't know how long the scraping of the clay should continue.

Did the potters in Bila village scrape without any measures at all? Certainly not-when we measured the thickness of the maximum diameter of the body of a standard earthenware, the capacity of about 1,500 to 2,000 cc, all of them fit within 6 to 7 


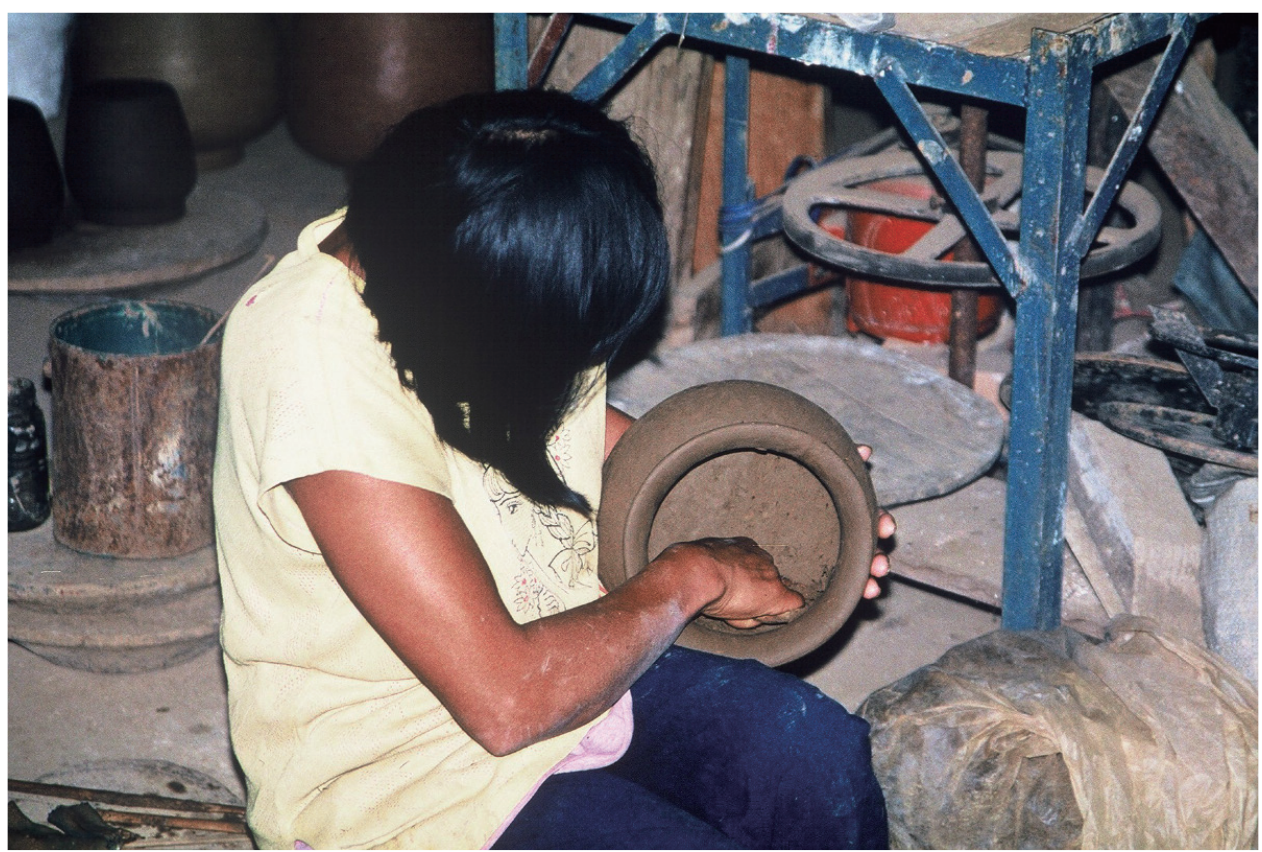

Fig. 2. Scraping the Inner Surface of Earthenware

mm (Fig. 3, Table 1). This result verified that the potters in Bila village were not working purposelessly, but were producing an even thickness. However, creating such an even thickness is a difficult task in reality. Even after learning the numerical thickness of the earthenware, I was still unable to determine the amount to cut the clay to reach the desired, uniform, thickness.

Therefore, naturally, I asked the local potters for guidance. The response was contrary to my expectations as I received ambiguous and vague descriptive answers such as, "until the thickness is appropriate." Numerical comprehension of thickness was unrecognizable for the potters in Bila Village. ${ }^{5}$ After consistent interviews, I concluded that such an equal thickness in each pottery was almost implicitly comprehended according to the experienced sensations that the potters built up, and this was agreed by them. If this is true, the even thickness of the pottery in Bila village is expressed as specific feelings gained through experiences (Ōnishi, 1998a, p. 480). In other words, it is not about the verbalization or the numerics, but the dependence on the empirically grasped sense or, in Michael Polanyi’s words, tacit knowledge (Polanyi, 1966). ${ }^{6}$ Hence, it is theorized that this even thickness is recognized by implicit and tacit knowledge. Yet,

\footnotetext{
${ }^{5}$ Indeed, this result should be anticipated. In fact, it should be considered unnatural to assume that the local potter recognizes the value of the pottery thickness in $\mathrm{mm}$ or $\mathrm{cm}$.

${ }^{6}$ Since tacit knowledge is a concept that exists inseparable from the actions of practice, it cannot be strictly identified by the discussion here alone. However, as described later, the knowledge of pottery making in Bila Village is also premised on the exercise of skills, so it can be regarded as almost synonymous with tacit knowledge.
} 

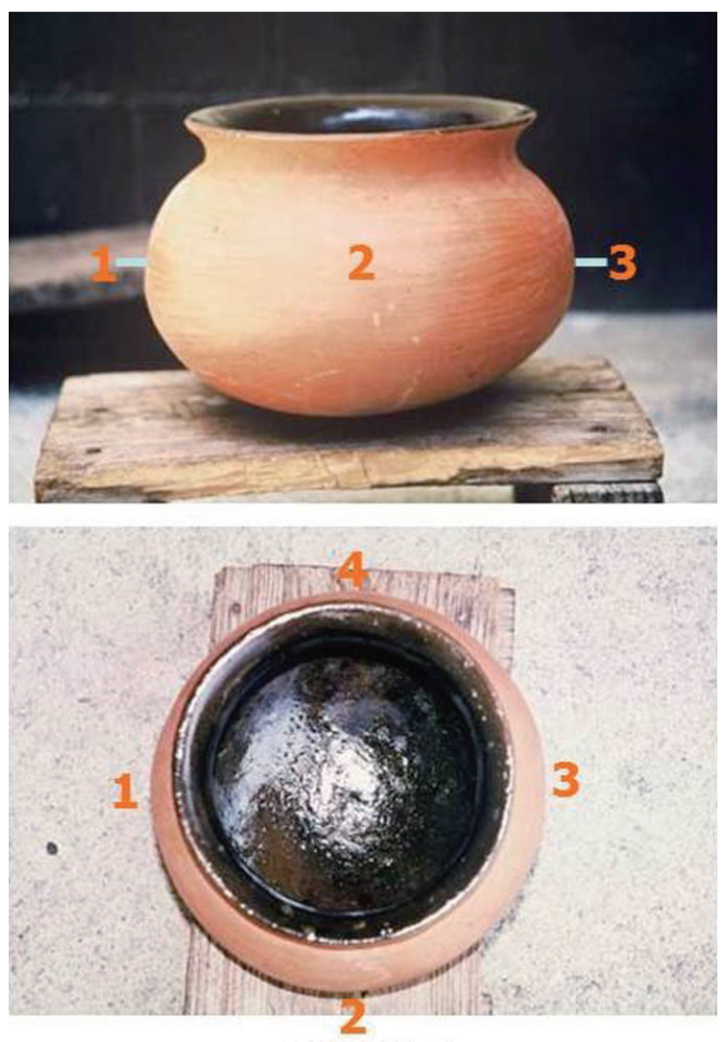

Fig. 3. Thickness of the Maximum Diameter of a Standard Earthenware Made by an Expert Note. Original information from Ōnishi (2014).

Table 1. Measurement Points of Fig. 3 With Compared Measurements of the Expert's and the Researcher's Earthenwares

\begin{tabular}{cll}
\hline Measurement Points & Expert & Researcher \\
\hline 1 & $7.0 \mathrm{~mm}$ & $6.5 \mathrm{~mm}$ \\
2 & $6.5 \mathrm{~mm}$ & $3.5 \mathrm{~mm}$ \\
3 & $6.0 \mathrm{~mm}$ & $5.0 \mathrm{~mm}$ \\
4 & $7.0 \mathrm{~mm}$ & $4.5 \mathrm{~mm}$ \\
\hline
\end{tabular}

Note. Original information from Ōnishi (2014).

a copious amount of knowledge is not enough to create this uniform thickness. This is because, the thickness value is only achieved through the movement of the body and tools - the techniques of the body (Sigaut, 1994, p. 439) is indispensable for the realization of this knowledge. 
Based on my experience, simple observation and interviews were not methods that helped in gaining such skills. This specific skill, of achieving uniformity of thickness, relies heavily on implicit and tacit knowledge that guides the determination, and the particular adjustments, in shaving off the clay. This determination and specific adjustments are techniques only learned through bodily experiences - they are embodied skills (Ōnishi, 1998a, p. 480). Thus, it is assumed that even thickness of pottery is identified by means of experience and tacit knowledge, and is maintained and reproduced through embodiment. It should be noted that such knowledge and skills are not limited to the scarping of clay but were found in various processes of pottery making in Bila Village: from the making of the base material to the burning (Ōnishi, 1998a, pp. 480-481).

Although, these knowledge and skills are not unique to pottery making in Bila Village. Similar nature is easily found in our daily lives, such as traditional craft productions. For example, imagine the balance needed for pedalling while sitting on the saddle of a bicycle or, the movement of the core of the body when skiing. These may help us understand the mediacy between the non-verbalized tacit/sensory actions of the body and embodied knowledge. Nonetheless, the knowledge and skills presented here cannot be separately distinguished - they are founded on each other and established as one. Again, it must be noted that these are aspects of knowledge and skills that are difficult to visualize and verbalize. It cannot be simply comprehended through observation and interviews: the foundations of ethnography. In addition, regardless of having these skills, a complete understanding and linguistic descriptions in these cases are close to impossible, just like the potters in Bila Village (Ōnishi, 1998b, pp. 2-4).

\section{Conclusion: Boundary Between Knowledge ANd SKILlS}

It has been demonstrated that the technical practice of craft production in the realm of folk culture in social sciences is mediated by the invisible and non-verbal tacit and experiential knowledge, recognized through embodiment. These knowledge and skills are not necessarily limited to non/pre-modern societies but are also found in contemporary societies. Although, there are questions of how far the two overlaps and interdepend on each other. This is because, founding on the discussions so far, knowledge relating to the actions of the body is inevitably indispensable to the support of skills. For example, when skiing, the ski's are aligned in parallel to the snow and by allowing the edge to be risen by tilting one's body opposite to the slope, the stability of speed as well as the shift of direction are controllable. In other words, any kind of knowledge related to human beings bodily practice on the physical world, although there are differences in intensity, skill exists as an essential requirement and is inseparably established. ${ }^{7}$

\footnotetext{
${ }^{7}$ In the case of skiing, tools such as skis and stocks are used, but they are only supplementary and it is the skier's body that makes the actions possible. As explained further, in modern society, with the industrial revolution innovating machine technology, cases appear where the human body support machines thus the body becoming a part of machines, for goal accomplishments. Immersed in this situation, skills as physical ability became infinitely unnecessary.
} 


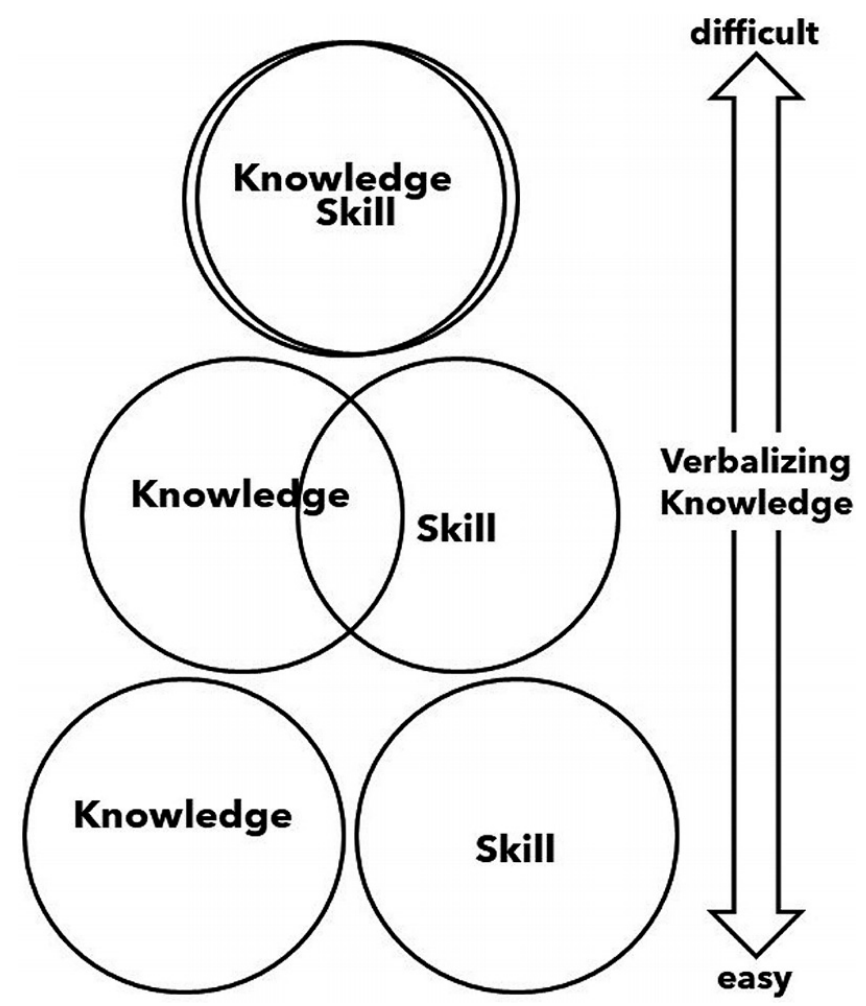

Fig. 4. Relationship With Knowledge and Skill Note. Original information from Ōnishi (2014).

However, some knowledge is not necessarily grounded on the premise of goal achievement through bodily practice. Some examples are: societal perspectives, religious systems, and mathematics - the existence of a Godly figure, the construct of the world, or evidence in mathematical propositions are questions structured on ideological or philosophical concepts. These are not strictly tied to goal achievement supported by skills. ${ }^{8}$ To rephrase, knowledge of worldview or religious beliefs exists on its own, unnecessary of bodily practice or even, performance in itself.

For this reason, knowledge and skills are intricately linked: they exist inseparably as one and at the same time, exist in complete independence (Fig. 4). On the other hand, this relationship between knowledge and skill provides an insight into the character and development stages of technology in society. This is easily understood by recalling the consequences of the Industrial Revolution in the modern era. Modern society has lived through technological innovations and industrial revolutions redeveloping work processes. What was once only possible by professionals were now accessible to anyone if they are

\footnotetext{
${ }^{8}$ However, world-views and belief systems can affect the activities of the real world-from the physical environment to social practice. For example, mathematics and physics have led to the development of nuclear weapons by being applied to science and technology.
} 
taught how to operate according to a manual. Although resisting to simplify, if I were to explain this history founded on the discussions thus far, technology that developed during the Industrial Revolution reconstructed the system of labor in contemporary societytasks were now simply fulfilled by knowing the operation of a machine. Professional skills were no longer mandatory in modern society - the Industrial Revolution divided knowledge from skills as far as possible, pushing forward divisions of labor. It was the advancement of mechanical technology that enabled labor without the expertise of professionals that lead to this segmentation.

This is not a historical event limited to modern society after the Industrial Revolution. For example, even before modern times, in the era of national and civilized society, pottery productions were established and contributed to the beginning of mass production. Due to productions, innovations such as potter's wheels were introduced and progressed a system of easy, rapid pottery production, only if produced following to the instructions. Of course, the operation of the wheel requires appropriate skills, however, the level of skills needed for the practice of knowledge was generally much lower than that of the technology prior to this system. This way, it can be argued that the separation of knowledge and skills also occurred in the non/pre-modern technology society. ${ }^{9}$ In a sense, the innovation of tools and machines in human history has promoted the separation of knowledge and skill and forwarded the generalization or universalization of technology.

\section{Perspective: Modern Society Founded by Verbalizing KnOWledge and Skills}

The discussions thus far argued, in modern society, the development of mechanical technology after the Industrial Revolution urged the separation of knowledge from skill. Certainly, this separation could have occurred in non/pre-modern societies, yet, its quality and quantity in modern society are incomparable, and undoubtedly, it is being pushed forward rapidly and extensively. This state is shaping our everyday world today.

The best example of this is manualized or verbalized technology. As pointed out in the previous section, this is nothing but the development of mechanical technology that separates knowledge from skill. If there is a machine that recognizes the purpose of labor, all that human beings are required is not to learn specific professional skills but, to grasp the systemized methods of production. These operation methods are nothing but the manual itself and, in most cases, are easily communicated. However, there are some machines that require intuition and tricks which are then interpreted as skills. Nonetheless, the modern society since the Industrial Revolution has aimed and, extensively promoted, the elimination of traditional mastery of skills, resulting to today where results are

\footnotetext{
${ }^{9}$ Considering these possibilities, the discussion in this paper seeks to clarify the cognitive behaviours of past human beings; not only in the contemporary sphere of psychology and cognitive science but also from excavated artefacts and archaeological sites. Therefore, this research has the potential to contribute to cognitive archaeology. In particular, ethnographic research on knowledge and skills of non/pre-modern societal human beings can highly anticipate new perspectives in exploring past cognitive behaviour, differing greatly in character and degree from research areas based on cases of modern society or laboratory data.
} 
obtained by the same procedure and operation from any person. This is a result of the flow of continuous innovation of mechanical technology.

To conclude, following the industrial revolution, modern society harnessed technological innovation which evolved to promoting the separation of knowledge and skills. As a result, a manualized and verbalized technology developed, driving out the skills of professionals. At the same time, this movement succeeded in turning society into an overwhelming amount of laborers. ${ }^{10}$ In that sense, exploring the relationship between knowledge and skills sprouts the significance of further research-to deepen the understanding of modern technology in contemporary society. Moreover, such reanalysis of knowledge and skills may contribute not only to the understanding of contemporary society but also the recognition of historical turning points such as civilization or state construct.

\section{CONFLICT OF INTEREST}

The author declares no conflicts of interest associated with this manuscript.

\section{REFRENCES}

Fukushima, M. (1993). Knowledge engineering in the wild: Introduction to the ethnography of tacit knowledge. Bulletin of the National Museum of Japanese History, 51, 11-44.

Fukushima, M. (Ed.). (1995). Shintai no kochikugaku [Studies on sociocultural construction of body]. Hituzi Syobo.

Ingold, T. (1993). Tool-use, sociality and intelligence. In K. R. Gibson \& T. Ingold (Eds.), Tools, language and cognition in human evolution (pp. 429-445). Cambridge University Press.

Lave, J., \& Wenger, E. (1991). Situated learning: Legitimate peripheral participation. Cambridge University Press.

Lemonnier, P. (1992). Elements for an anthropology of technology. The University of Michigan Press. https://doi.org/10.3998/mpub.11396246

Lock, M. (1993). Cultivating the body: Anthropology and epistemologies of bodily practice and knowledge. Annual Review of Anthropology, 22, 133-155. https://doi.org/10.1146/annurev.an.22.100193.001025

Mauss, M. (1934). Les techniques du corps. Journal de Psychologie, 32(3-4), 271-293.

Ōnishi, H. (1998a). The birth of a Kankana-ey potter: Practice and learning of a folk technique among Kankana-ey society. Japanese Journal of Ethonology, 62(4), 470-493. https://doi.org/10.14890/ minkennewseries.62.4_470

Ōnishi, H. (1998b). Ruson-to hokubu Kankana-ey shakai nioite katachizukurareta dokiseisakusha no shintai [The potters' body of the Kankana-ey society of Northern Luzon]. Material Culture: Journal of Archaeologico-Folkloric Studies, 64, 1-28.

Ōnishi, H. (2014). Gijutsu to shintai no minzokushi: Philippine Luzon-to sanchimin shakai ni ikizuku minzokukogei [An ethnography of technology and body: Folk craft in the Philippine highlanders'

${ }^{10}$ The development of mechanical technology since the Industrial Revolution has reduced the value of the professional skills and reconstructed society. Women and children without special skills were made into a reserve army of workers. Modern technology, with mechanical technology as a representative, has fixed the overwhelming majority of social members to workers: those engaged in production as employees with no capital or skills. This was the biggest factor in revitalizing the unequal power relationship with capitalists. 
society of Northern Luzon]. Showado.

Polanyi, M. (1966). The tacit dimension. Routledge.

Polanyi, M. (1969). Knowing and being: Essays by Michael Polanyi (M. Grene, Ed.). Routledge.

Sigaut, F. (1994). Technology. In T. Ingold (Ed.), Companion encyclopedia of anthropology: Humanity, culture and social life (pp. 420-459). Routledge.

Tanabe, S. (1989). Jinruigakuteki ninchi no boken: Ideorogi to purakuteisu [Adventure of anthropological cognition: Ideology and practice]. Dobunkan.

Tanabe, S. (2002). The concept of practice in reflexive anthropology: On Bourdieu's habitus and beyond. Bulletin of the National Museum of Ethnology, 26(4), 533-573. https://doi.org/10.15021/00004050

Tanabe, S., \& Matsuda, M. (Eds.). (2002). Nichijoteki jissen no esunogurafi: Katari, komyunitei, aidenteitei [Ethnographies of daily practice: Narrative, community, and identity]. Sekaishisosha.

(Manuscript received 4 February, 2021; Revision accepted 12 April, 2021; Released online in J-STAGE as advance publication 23 June, 2021) 